\title{
Engineered Fluorescent Proteins Bring Biochemistry To Light
}

\author{
Robert E. Campbell \\ Department of Chemistry, University of Alberta, Edmonton, Alberta, T6G 2G2 CANADA
}

To ensure the continued advancement of cell biology, it is critical that the visualization of biochemical activities in live model animals with high temporal and spatial resolution ceases to be a technical tour de force, and instead becomes a routine and widely accessible endeavor. In the last decade a convergence of interests between physicists, chemists, biologists, and engineers has resulted in tremendous progress towards achieving this goal. Accordingly, the research community is moving towards a "neurophotonic" future in which minimally invasive optical techniques fully replace traditional biochemical methods for the study of cell physiology.

Arguably, the most significant advancements of the past decade have been in the area of optogenetic reporters and actuators [1]. The term "optogenetic" refers to fully genetically encoded proteins that either fluoresce (reporters), or undergo a change in function (actuators), upon absorption of visible light. As they are genetically encoded, optogenetic tools are fully compatible with viral delivery methods, and the construction of transgenic model organisms that dramatically facilitate the ability of researchers to non-invasively induce and visualize biochemical activities.

The Campbell research group is focused on the use of protein engineering for the development of new and improved optogenetic tools [2,3]. Our most recent work has been directed towards the development of red fluorescent $\mathrm{Ca}^{2+}$ indicators, and in this seminar I will describe a variety of new probes that are optimized for 2-photon excitation and use in high $\left[\mathrm{Ca}^{2+}\right]$ environments. I will also describe our successful efforts to engineer a new generation of red fluorescent voltage reporters and how we are now using similar engineering efforts to make reporters for neurotransmitters.

\section{References:}

[1] Alford, S.C., Wu, J., Zhao, Y., Campbell, R.E. \& Knöpfel, T., Biol. Cell 105 (2013), p. 14.

[2] Zhao, Y. et al, Science 333 (2011), p. 1888.

[3] Wu, J. et al, ACS Chem. Neurosci. 4 (2013), p. 963.

[4] The authors acknowledge funding from Alberta Innovates, NSERC, and CIHR. 


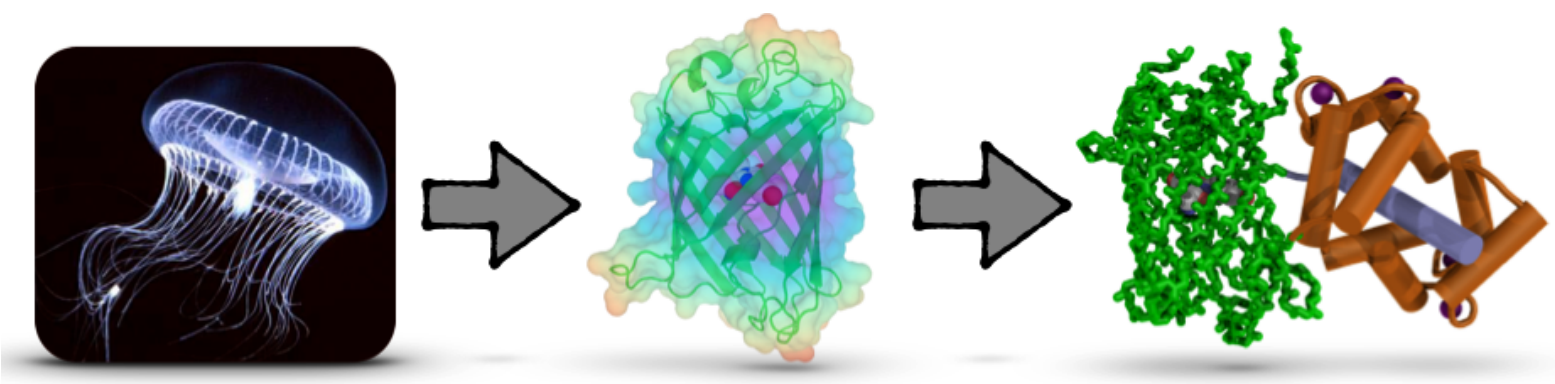

Figure 1. Naturally occurring fluorescent proteins from marine organisms are engineered to be useful fluorophores for live cell imaging. With extensive engineering, these fluorophores can be converted into optogenetic reporters of biochemical activities. 